\title{
An Improved MOM-GEC Method for Fast and Accurate Analysis of 2-D Planar Structures in Waveguides: Application to Planar Microstrip Antennas
}

\author{
N. Oueslati ${ }^{1}$ and T. Aguili ${ }^{2}$ \\ SysCom laboratory, National Engineering School of Tunis B.P. 37 Le Belvedere, Tunis 1002, Tunisia
}

\begin{abstract}
This paper presents a new hybridization between MoM-GEC and a MultiResolution analysis (MR) which is based on the use of wavelets functions as trial functions. The proposed approach is developed to speed up convergence, alleviate calculation and then provide a considerable gain in requirements (processing time and memory storage) because it generates a sparse linear system. The approach consists in calculating the total current and input impedance on an invariant metallic pattern through two steps. The first one consists in expressing the boundary conditions of the unknown electromagnetic current with a single electrical circuit using the Generalized Equivalent Circuit method (GEC) and then deduce an electromagnetic equation based on the impedance operator [1,2]. The impedance operator used here is described using the local modal basis of the waveguide enclosing the studied structure. The second step consists in approximating the total current using orthonormal periodic wavelets as testing functions and the local modal basis of the waveguide as basis functions.The proposed approach allows fast calculation of such inner products through the use of the wavelets multiresolution (MR) analysis advantages, thus significantly reducing the required CPU-time for microstriptype structure analysis [3,4]. A sparse matrix is generated from the application of a threshold.A sparsely filled matrix is easier to store and invert [5,6]. Based on this approach, we study a 2-D planar structure including a step discontinuity. The obtained results show good accuracy with the method of moments. Moreover, we prove the considerable improvements in CPU time and memory storage achieved by the MR-GEC approach when studying these structures.
\end{abstract}

\section{Introduction}

Recently, almost planar structures in 2D-dimensional case become the subject of important scientific research, in particular in defense and space applications, communication systems and electronics devices such as: phased array radar systems, Frequency selective surfaces (FSSs) applications, [7,8]. Many numerical techniques have been invested in this context. Most of these methods consider the radiating structures opened (not shielded).

All the publications in this domain $[9,10]$ have shown that the integral formulation, specifically the moment method, was the best to set the full wave methods more efficiently. The method of moments uses the integral from of Maxwell's equations. These equations are solved by the finite linear space approximation which leads to a matrix equation of which the rank is proportional to the number of unknowns. However, in most cases, knowledge of the Green's functions, either in simple functional form or series expansion form, are required. This limits the use of MoM to simple structures in which Green's functions are available. The integral equation needs enormous amount of analytical effort to implement when supports of basis and testing functions are overlapping or share some common points. On the other hand, different formulation are needed when the structure changes. Indeed, the application of the spatial-domain MOM requires the necessary Green's functions in the spatial domain that can be obtained from their spectral domain counterparts, therefor, additional numerical implementation are needed.

Hence, the investigation of complex structures poses a major problem due to the limitation of MoM. Thus, it is necessary to use the hybridization of numerical methods to overcome the problem of modeling complex structures that contain fine details in large domains. Indeed, the hybridization of numerical methods is one of speediest, efficient and accurate solutions of electromagnetic modeling of complex structures. For example, hybridization of analytical solution and MoM has been investigated in $[11,12]$. In this paper, the method of moment is combined to the Generalized Equivalent Circuit (MoM-GEC) to convert an integral or differential equation into a linear system that will be solved using a matrix representation. The equivalent circuits have been introduced in the development of integral methods formulation with equivalent circuit problems $(V, I)$ instead of field problems $(E, H)$. This constitutes the concept of MoM-GEC. Its key idea is the transposition of field problems in GEC which are simpler to treat and the use of the impedance (admittance) operator simplifying then the transition between spectral and spatial domains. 
A major feature of this method is reducing the spatial degree size of problems. The study of a volume structure is solved by a surface approach which makes this method particularly suitable for finite areas in infinite medium. Determining the electromagnetic field radiated by an object in a certain volume of calculation is reduced to determine the current on its surface, and this problem requires a surface meshing and not a volume one, so that we can find the electromagnetic field radiated by the structure in the entire space. MoM-GEC is a general method that transforms a functional (differential or integral equation) into a linear equations system that can be solved by matrix techniques.

The procedure of the MoM-GEC method is based on minimizing the residual error on basis and test functions in order to get the convergence of the solution. As the number of test functions is high, we need a very high number of basis functions to get convergence [2]. This leads to manipulating matrices with great sizes. Consequently, the needed memory resources and computational time to solve such problems will be considerably increased.

In this context, several fast algorithms have been used to reduce the computational complexity and memory requirement, such as Finite Element Method (FEM), which formulate the electromagnetic problem using differential equation, And more power method such as the Fast Multi-pole Method or the Multilevel Fast Multi-pole Algorithm (MLFMA), which need more powerful machine to be implemented. In contrast, WaveletBased Moment method which can be implemented easily in personal computer [13,14].

the approach proposed in this paper uses Galerkin's procedure, leading to a sparse matrix, which elements are constituted of inner products of the local modal basis of the waveguide as basis functions, with periodic wavelets as trial functions obtained by integral calculation.

In this paper, we apply the principle of MR-GEC to analyze planarmicrostrip antenna in waveguide. The remainder of the paper is organized as follows: Section 2 describes the generalized equivalent circuits' concept. The choice of wavelets as trial functions is described in Section 3. The MR-GEC method will be applied in Section 4 to a planar microstripantenna and the theoretical formulation is presented. Section 5 illustrates the numerical results and discussions at the convergence for the input impedance viewed by located source and the reflection coefficient, and compared to advanced design system (ADS) and SONNET.

\section{Principles Of GEC Method}

The equivalent circuit presents a true electric image of the studied structures for describing the discontinuity and its environment. In fact, for alleviating the resolution of Maxwell's equations, the method of Generalized Equivalent Circuit (MGEC) was proposed [1,2] in order to represent integral equations by equivalent circuits that express the unknown electromagnetic boundary conditions. The discontinuity environment is expressed by an impedance operator or admittance operator that represents boundary conditions on each side of discontinuity surface.

In the discontinuity plane, the electromagnetic state is described by generalized test functions that are modeled by virtual sources not storing energy. The discontinuity environment is expressed by an impedance operator or admittance operator that represents boundary conditions on each side of discontinuity surface. However, the wave exciting the discontinuity surface is represented by a real field source or a real current source because it delivers energy.

Generally, the electromagnetic modeling with GEC extends the Kirchhoff's laws used in $(V, I)$ concept to the Maxwell's formalism $(E, H)$. In order to apply Kirchhoff's laws accurately, we should substitute the magnetic field by the current density $J$ defined as $\vec{J}=\vec{H} \wedge \vec{n}$ where $\vec{n}$ is the normal vector to the discontinuity surface. It is noted that these generalized equivalent circuits are associated to perfect interfaces, which are characterized by the fact that electric field and current density are defined on complementary domains.

\subsection{The adjustable virtual sources}

Let's consider $D$ a discontinuity plane formed by metallic and dielectric patterns $(D=D M+D D)$. Based on current and field properties on $D, D M$ is the metallic sub-domain on which the field is null, and its dual current is not null. However, $D D$ is the dielectric domain on which the current is null, and its dual field is not null. The concept of virtual source is to assemble all field and current representations in an only one which will be valid in all points of the domain $D$. Figure 1(a) and Figure 1(b) describes virtual sources.
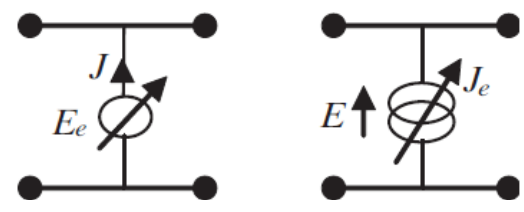

Figure 1.Symbolic notation of virtual sources: (a) field source; (b) current source. 


\subsection{The impedance operator}

The impedance operator, as shown in Figure 2 is a modal integro-differential operator and presents an alternative to the Green operator in the spectral field. The relation between $\vec{E}$ and $\vec{J}$ classically takes the form of the Ohm's law: $\vec{E}=\hat{Z} \vec{J}$ or $\vec{J}=\hat{Y} \vec{E}$.

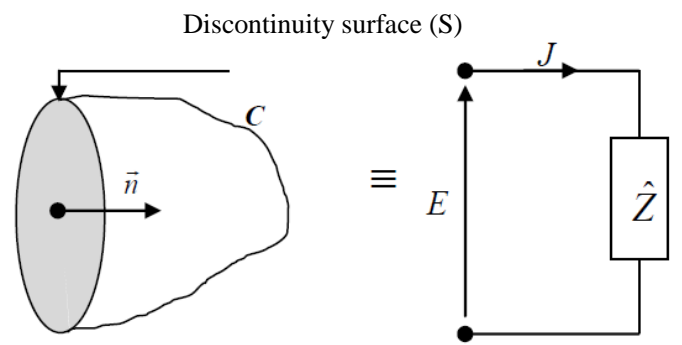

Figure 2.Equivalence between the half-

\subsection{The excitation sources}

The excitation sources are defined on a small surface $(S)$ and are characterized by a quasi-static voltage or current. We can distinguish two types of planar localized sources: the localized field and current sources. Each of the two sources is described in Figure 3.

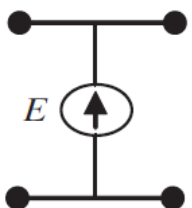

(a)

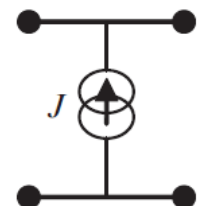

(b)

Figure 3. (a) field source, (b) current source.

\section{Choice of trial functions: wavelets expansion}

In this paper, the trial functions are presented as a superposition of wavelets at several scales including the scaling function. A Galerkin method is then applied, where the set of trial functions are used as weighting functions to transform the integral equation into algebraic equations in the expansion coefficients.

Basic wavelet theory can be found in many excellent books $[15,16]$. The wavelet theory can be extended to any size. We briefly present the case in two dimensions (2D), since we are dealing with twodimensional structures. There are several approaches for increasing wavelet transform defined in the onedimensional case to the two-dimensional case. We will limit our work to the study of the separable dyadic multiresolution analysis presented by Mallat [16].

Consider a set of subspaces $\left(V_{s}\right)_{s \in Z}$ in $L^{2}\left(R^{2}\right)$.

Each sub-space $V_{s}$ at resolution $2^{-s}$ is constructed by the Cartesian product of two dimensional AMR at the same level of resolution, that is to say

$$
V_{s, k_{x}, k_{y}}=V_{s, k_{x}} \otimes V_{s, k_{y}}
$$

An orthonormal base $V_{s}$ is composed of scales functions defined:

$$
\phi_{s, k_{x}, k_{y}}(x, y)=\phi_{s, k_{x}}(x) \phi_{s, k_{y}}(y) ; k_{x}, k_{y} \in \mathrm{Z}
$$

Where $s$ is the level of resolution, the index $k_{x}$ represents the expansion of the subspace associated with the variable $\boldsymbol{x}$ and $k_{y}$ corresponds to the expansion of the subspace associated with the variable $\boldsymbol{y}$. These two subspaces are generated by two functions at different scales:

$$
\begin{aligned}
& \phi_{s, k_{x}}(x)=2^{s / 2} \phi\left(2^{s} x-k_{x}\right) \mapsto V_{s}^{x} \\
& \phi_{s_{k_{y}}}(y)=2^{s / 2} \phi\left(2^{s} y-k_{y}\right) \mapsto V_{s}^{y}
\end{aligned}
$$


Similarly, the subspaces generated by the wavelet are noted:

$$
\begin{gathered}
\psi_{s,_{k_{x}}}(x)=2^{s / 2} \psi\left(2^{s} x-k_{x}\right) \mapsto W_{s}^{x} \\
\psi_{s_{k_{y}}}(y)=2^{s / 2} \psi\left(2^{s} y-k_{y}\right) \mapsto W_{s}^{y}
\end{gathered}
$$

The dimensional subspace $W_{s}$ is the orthogonal complement of $V_{s}$ in $V_{s+1}$.

The properties of the multi-resolution analysis and the distributivity of the tensor product used to write:

$$
\begin{aligned}
& V_{s, k_{x}, k_{y}}=V_{s}^{x} \otimes V_{s}^{y} \\
& =\left(V_{s-1}^{x} \oplus W_{s-1}^{x}\right) \otimes\left(V_{s-1}^{y} \oplus W_{s-1}^{y}\right) \\
& =\left(V_{s-1}^{x} \otimes V_{s-1}^{y}\right) \oplus\left(V_{s-1}^{x} \otimes W_{s-1}^{y}\right) \oplus\left(W_{s-1}^{x} \otimes V_{s-1}^{y}\right) \oplus\left(W_{s-1}^{x} \otimes W_{s-1}^{y}\right) \\
& =V_{s-1} \oplus W_{s-1}^{h} \oplus W_{s-1}^{v} \oplus W_{s-1}^{d}
\end{aligned}
$$

This equation shows four different sub-spaces associated with the two-dimensional multi-resolution analysis.

Each of these sub-space is generated by a mother function of its own:

$$
\begin{aligned}
& \phi_{s, k_{x}, k_{y}}(x, y)=\phi_{s, k_{x}}(x) \phi_{s, k_{y}}(y) \mapsto V_{s} \\
& \psi_{s, k_{x}, k_{y}}^{h}(x, y)=\phi_{s, k_{x}}(x) \psi_{s, k_{y}}(y) \mapsto W_{s}^{h} \\
& \psi_{s, k_{x}, k_{y}}^{v}(x, y)=\psi_{s, k_{x}}(x) \phi_{s, k_{y}}(y) \mapsto W_{s}^{v} \\
& \psi_{s, k_{x}, k_{y}}^{d}(x, y)=\psi_{s, k_{x}}(x) \psi_{s, k_{y}}(y) \mapsto W_{s}^{d}
\end{aligned}
$$

It is therefore necessary to generate a two-dimensional multiresolution analysis, four different functions. The indices $\mathrm{h}, \mathrm{v}$ and associated with new wavelets are given respectively for horizontal, vertical and diagonal. This terminology is taken from [17]. They highlight the main direction of oscillation of the signal.

With these notations, the approximation of a function $f(x, y)$ of $L^{2}\left(R^{2}\right)$ at the resolution $2^{-s}$ can be expressed in terms of projections on the subspaces $V_{s}$ by:

$$
P_{s}(f)=\sum_{k_{x} \in \mathrm{Z}} \sum_{k_{y} \in \mathrm{Z}}\left\langle f \mid \phi_{s, k_{x}, k_{y}}\right\rangle \phi_{s, k_{x}, k_{y}}(x, y)
$$

Then the improved approximation of the function $\mathrm{f}$ at a finer level of resolution $2^{-s-1}$ includes information generated by three subspaces details. One can then write:

$$
P_{s+1}(f)=P_{s}(f)+\sum_{i=h, v, d} \sum_{k_{x} \in Z} \sum_{k_{y} \in Z}\left\langle f \mid \psi_{s, k_{x}, k_{y}}^{i}\right\rangle \psi_{s, k_{x}, k_{y}}^{i}(x, y)
$$

The development of a function defined on a finite surface assumes the same shape but with finite limits for translation indexes $k_{x}$ and $k_{y}$.

The MALLAT decomposition algorithm in the two-dimensional case is a direct extension of the onedimensional versions. In the $2 \mathrm{D}$ case, we have four types of coefficients defined by:

$$
\begin{array}{r}
c_{s, k_{x}, k_{y}}=\left\langle f \mid \phi_{s, k_{x}, k_{y}}\right\rangle=\int_{-\infty-\infty}^{+\infty} \int_{-\infty}^{+\infty} f(x, y) \phi_{s, k_{x}, k_{y}}(x, y) d x d y \\
d_{s, k_{x}, k_{y}}^{i}=\left\langle f \mid \psi_{s, k_{x}, k_{y}}^{i}(x, y)\right\rangle=\int_{-\infty-\infty}^{+\infty} \int_{-\infty}^{+\infty} f(x, y) \psi_{s, k_{x}, k_{y}}^{i}(x, y) d x d y ; i=h, v, d
\end{array}
$$

The mathematical equations that generate the 2D-DWT are given by: 


$$
\begin{aligned}
& c_{s, k_{x}, k_{y}}=\sum_{k_{x} \in Z k_{y} \in Z} h_{n_{x}-2 k_{x}} h_{n_{y}-2 k_{y}} c_{s+1, n_{x}, n_{y}} \\
& d_{s, k_{x}, k_{y}}^{h}=\sum_{k_{x} \in Z k_{y} \in Z} h_{n_{x}-2 k_{x}} g_{n_{y}-2 k_{y}} c_{s+1, n_{x}, n_{y}} \\
& d_{s, k_{x}, k_{y}}^{v}=\sum_{k_{x} \in Z k_{y} \in Z} g_{n_{x}-2 k_{x}} h_{n_{y}-2 k_{y}} c_{s+1, n_{x}, n_{y}} \\
& d_{s, k_{x}, k_{y}}^{d}=\sum_{k_{x} \in Z k_{y} \in Z} g_{n_{x}-2 k_{x}} g_{n_{y}-2 k_{y}} c_{s+1, n_{x}, n_{y}}
\end{aligned}
$$

So, for calculating the scales coefficients (approximation) and wavelet coefficients (details) at multiple resolution levels, we simply calculate scales coefficients at the finest resolution and then deduce by the MALLAT's algorithm other approximation coefficients and details.For finite-size bodies, the sources domain of definition is inherently finite, and the difficulties articulated are encountered if a multiresolution approach is sought. In literature, many solutions are provided, and in this work, to expand the current on the fundamental interval, the use of a periodic multiresolution system is kept. Such a system can be derived from a conventional multiresolution one by the periodic extension.

\subsection{Formulation of the MR-GEC}

\section{Validation of numerical results}

The microstrip STEP is widely appearing in low pass filters and other microwave circuits [18]. Therefore, it is important to develop analytical techniques to compute accurately the characteristics of this discontinuity. The STEP discontinuity structure to be studied is a rectangular microstripantenna, as described in Figure 1, laid on an isotropic dielectric substrate and excited on the plane of the circuit by a localized voltage source. This structure is shielded in a rectangular metallic box with electric walls and which the top cover is placed endlessly. We study this structure at $\mathrm{f}=4.9 \mathrm{GHz}$.

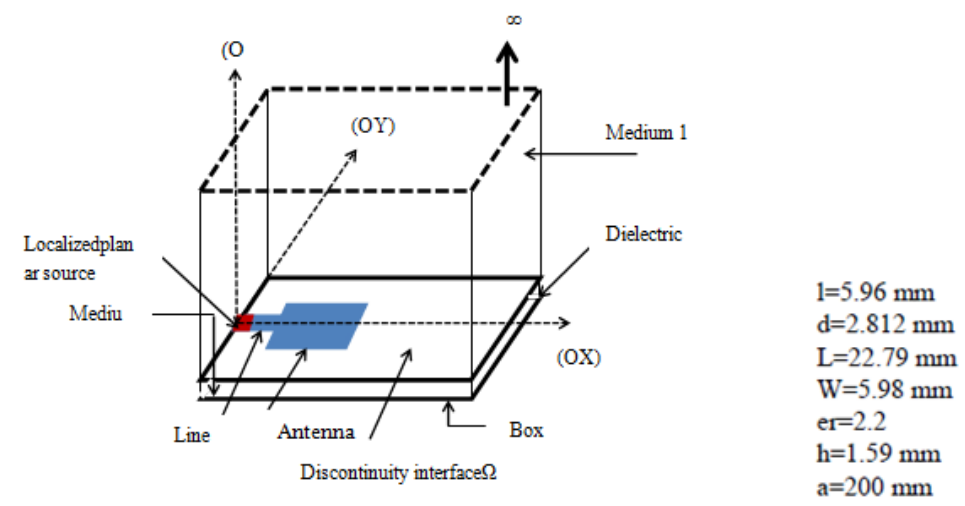

Figure 4.Characteristics of the rectangular microstrip antenna.

Based on the implementing rules, we can formulate the problem of searching the current $J$ flowing in the structure by studying its equivalent circuit. To do this, we bring back the different modes propagating in space on the study area and we transpose vacuum to a dipole representing the admittance of vacuum $\widehat{Y}_{1}$. The same applies to the ground plane which is replaced by a dipole representative of the admittance shorted $\widehat{Y}_{2}$. We use an arbitrary excitation $\overrightarrow{E_{0}}$ on the microstrip line subregion. On the plane of the strip, the current density on the metal region $\overrightarrow{J_{M}}$ is expressed in term of trial functions basis. We then obtain the circuit of Figure 5.

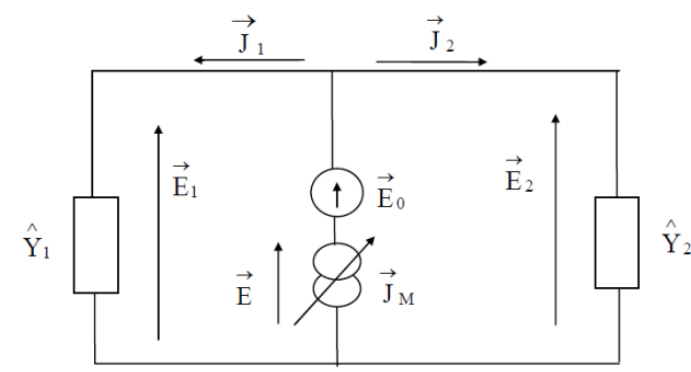

Figure 5.Equivalent circuit of the microstrip line. 
Lets $\left\{f_{m n}{ }^{T E, T M}\right\}$ be the local modal basis of the EEEEE waveguide enclosing the microstrippatch antenna [1].

$\vec{E}_{0}=V_{0} \vec{G}(x, y)$ denotes the electric field excitation, defined on the surface of the planar source according to a distribution $\vec{G}$ on a sub-region of the microstrip line and with an amplitude $V_{0}$.

$J_{M}$ can be expressed in terms of the magnetic fields defined in the discontinuity plane as [19]:

$$
\vec{J}_{M}=\vec{H}^{1} \wedge \vec{n}^{1}+\vec{H}^{2} \wedge \vec{n}^{2}=\vec{J}_{1}+\vec{J}_{2}
$$

where $\vec{n}^{1}$ and $\vec{n}^{2}$ indicate unit vectors normal to the discontinuity plane and directed toward $z>0$ and $z<0$, respectively. Here, the admittance operators $\hat{Y}_{1}$ and $\hat{Y}_{2}$ for region 1 and 2 , respectively, are viewed by the discontinuity plane.

Figure 5 describes the electric circuit obtained by the application of GEC modeling to the studied structure, the generalized Ohm and Kirchhoff laws are then rewritten as equations system:

$$
\left\{\begin{array}{c}
E_{0}+E_{2}=\hat{\mathrm{Y}}_{2}^{-1} J_{2} \\
E_{0}+E_{1}=\hat{\mathrm{Y}}_{1}^{-1} J_{1} \\
J_{1}+J_{2}=J_{M}
\end{array}\right.
$$

We obtain then the following system:

$$
\left\{\begin{array}{c}
J_{M}=J_{1}+J_{2}=J \\
E=-E_{0}+\left(\hat{Y}_{1}+\hat{Y}_{2}\right)^{-1} J_{M}=-E_{0}+\hat{Z} J_{M}
\end{array}\right.
$$

Sources and their duals are related as:

$$
\left(\begin{array}{c}
J \\
E_{e}
\end{array}\right)=\left(\begin{array}{cc}
0 & 1 \\
-1 & \hat{Z}
\end{array}\right)\left(\begin{array}{c}
E_{0} \\
J_{e}
\end{array}\right)
$$

This method requires the involvement of a complete set of orthogonal basis functions $\left\{\left|f_{m, n}\right\rangle\right\}_{m, n=0, N}$, with $\mathrm{N}$ the number of modes), which should satisfy the boundary conditions imposed by the shielding [19]. This process also needs to calculate the mode impedances $z_{m n}{ }^{1}$ and $z_{m n}{ }^{2}$, of region 1 and 2, respectively, at the discontinuity plane. For each medium $i \in\{1,2\}$, t2he expression of the total modal admittance and impedance for $T E_{m, n}$ and $T M_{m, n}$ modes are given respectively by:

$$
\left\{\begin{array} { c } 
{ T E : y _ { m , n } ^ { i , T E } = \frac { \gamma _ { m , n } ^ { i } } { j \omega \mu _ { 0 } } } \\
{ T M : y _ { m , n } ^ { i , T M } = \frac { j \omega \varepsilon _ { 0 } } { \gamma _ { m , n } ^ { i } } }
\end{array} \Rightarrow \left\{\begin{array}{c}
T E: z_{m, n}^{i, T E}=\frac{1}{y_{m, n}^{i, T E}} \\
T M: z_{m, n}^{i, T M}=\frac{1}{y_{m, n}^{i, T M}}
\end{array}\right.\right.
$$

$$
\gamma_{m, n}^{i}=\sqrt{\left(\frac{m \Pi}{a}\right)^{2}+\left(\frac{n \Pi}{b}\right)^{2}-k_{i}^{2}}
$$

with $k_{i}^{2}=\left\{\begin{array}{c}k_{0}^{2} ;(i=1(\text { vacuum })) \\ k_{0}^{2} \varepsilon_{r} ;(i=2(\text { dielectric })\end{array}\right.$

When the structure along the $z$ axis is terminated in the metallic wall (short circuit), the admittance seen by each mode at the interface is given by:

$$
Y_{m, n}^{\alpha, i}=y_{m, n}^{\alpha, i} \operatorname{coth}\left(\gamma_{m, n}^{i} h_{i}\right),
$$

$h_{i}$ is the thickness of the medium $i$, in our case, $i=2 ; h_{i}=h$ (the medium is the dielectric).

If a termination was an open circuit (no metallic wall at the end of the medium $i$ ), the admittance seen by each mode at the interface is given by:

$$
Y_{m, n}^{\alpha, i}=y_{m, n}^{\alpha, i}
$$

In our case, the medium $i$ is the vacuum.

The total impedance seen by each mode at the interface is given by: 


$$
Z_{m, n}^{\alpha}=\frac{1}{Y_{m, n}^{\alpha}}=\frac{1}{Y_{m, n}^{\alpha, 1}+Y_{m, n}^{\alpha, 2}} ; \alpha \in\{T E, T M\}
$$

The expression of the impedance operator is expressed as:

$$
\hat{\mathrm{Z}}=\sum_{m, n}\left|f_{m, n}\right\rangle z_{m, n}\left\langle f_{m, n}\left|=\sum_{m, n}\right| f_{m, n}^{T E}\right\rangle z_{m, n}^{T E}\left\langle f_{m, n}^{T E}\left|+\sum_{m, n}\right| f_{m, n}^{T M}\right\rangle z_{m, n}^{T M}\left\langle f_{m, n}^{T M}\right|
$$

At this stage, we can project the unknown $\overrightarrow{J_{M}}$ (current) on the basis of trial functions, so we will express it with series of scaling and wavelets functions (test functions) and then write [13]:

$J(x, y)=\sum_{k_{x}=0}^{2^{s(-)}} \sum_{k_{y}=0}^{-1} c_{s(-), k_{x}, k_{y}} \phi_{s(-), k_{x}, k_{y}}^{i(-)}(x, y)+\sum_{s=s(-)}^{s(+)} \sum_{i=h, v, d} \sum_{k_{x}=0}^{2^{s(+)}-1} \sum_{k_{y}=0}^{2^{s(+)}-1} d_{s, k_{x}, k_{y}}^{i} \psi_{s, k_{x}, k_{y}}^{i}(x, y)(20)$

wheres $(-)$ is the coarsest level and $s(+)$ the finest level.

We use the Galerkin method to solve the Eq.20 numerically. The method consists in determining the system matrix from the equivalent circuit, and make projections based on test functions. The resulting matrix equation is written in this form:

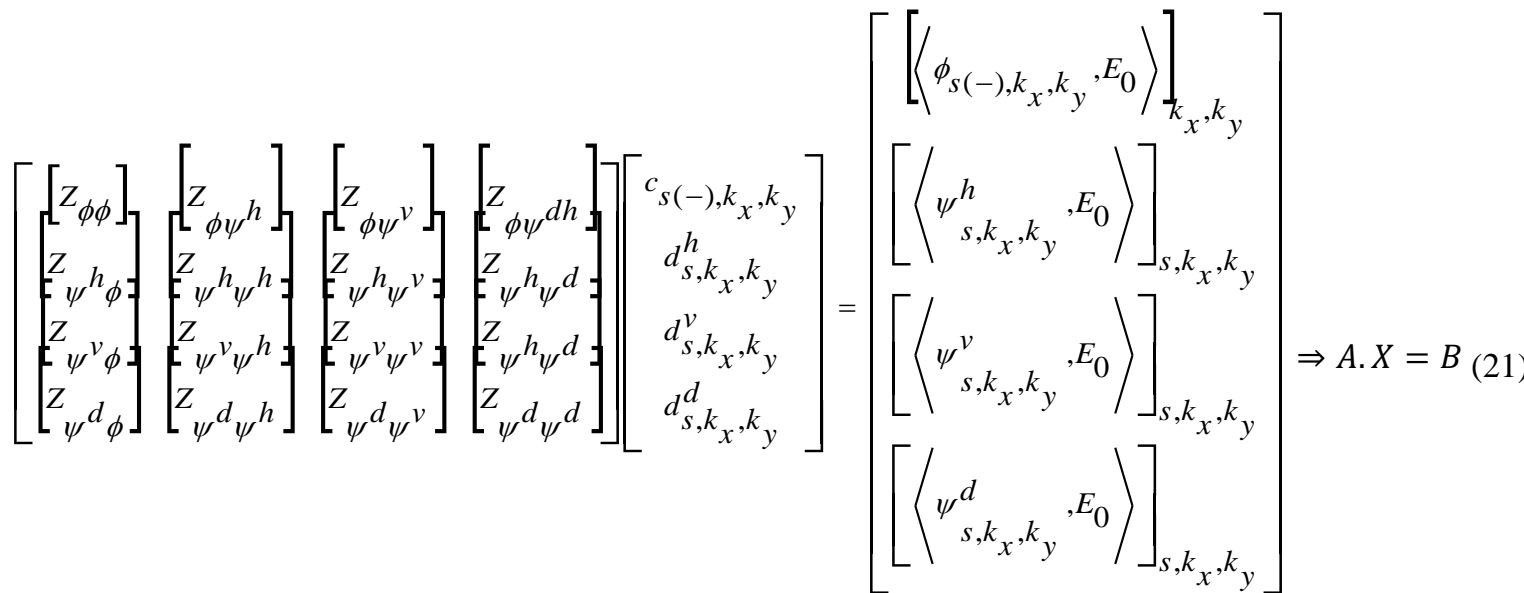

In practice, a wavelet with $\mathrm{N}$ vanishing moments enables the cancellation of all wavelet coefficients of a polynomial signal whose degree is less than $\mathrm{N}$. Thus, if is polynomial of degree less than $\mathrm{N}$ on the support of $\psi_{s, k_{x}, k_{y}}^{i}$, then $d_{s, k_{x}, k_{y}}^{i}=\left\langle f \mid \psi_{s, k_{x}, k_{y}}^{i}(x, y)\right\rangle \geq 0$ [19]. This result is quite significant because it enables high compression rates (many wavelet coefficients are zero or negligible). If the $f_{m, n}^{T E}$ and $f_{m, n}^{T M}$ modes of the empty waveguide are smooth enough to be approximated by a polynomial expression of order less than $\mathrm{N}$, then the elements of matrix A vanishes or becomes very small. A threshold is adjusted to get a minimal error in the current density.

A compression rate is defined as follows:

$$
T C(\%)=\frac{\text { numberofmatrixelementssettozero }}{(\text { numberoftrialfunctions })^{2}} * 100
$$

The input impedance seen by the source is determined by:

$$
Z_{\text {in }}=\frac{\left\langle E_{0} \mid E_{0}\right\rangle}{\left\langle E_{0} \mid V_{M}\right\rangle}=\left(B^{T} A^{-1} B\right)^{-1}
$$

The reflection coefficient is then expressed as:

$$
S_{11}=\frac{Z_{\text {in }}-Z_{c}}{Z_{\text {in }}+Z_{c}}
$$

where $Z_{C}=50 \Omega$ is also the characteristic impedance of microstrip line.

\subsection{Validation of the analysis method}

We used the 'Daubechies 5' family on the line and the 'Coifman 5' family on the radiator. Convergence is DOI: $10.9790 / 1676-1104021424$ 
reached to a level of resolution $s_{-}$line $=3$ and $s_{-}$patch $=3$. The number of convergence to basic functions is 40000 (200x200) TE and TM modes.

At the frequency $\mathrm{F}=4.9 \mathrm{GHz}$, the value of the input impedance calculated at convergence is presented in Table 1. A good agreement between the values found is approved.

Table1.Comparison of Zin of the rectangular patch antenna at convergence.

\begin{tabular}{|l|l|l|l|}
\hline & SONNET & MoM_GEC +wavelets & MoM_GEC +sinusoidals \\
\hline Zin & $4.34-39.12 \mathrm{i}$ & $3.96-38.025 \mathrm{i}$ & $5.17-42.16 \mathrm{i}$ \\
\hline
\end{tabular}

Figure 6 represents the compression ratio depending on the $\delta$ precision for both types of test functions. We can see that with the approach using sinusoidal functions the compression ratio does not exceed $45 \%$ for accuracy $\delta=3.510^{-6}$ and stabilizes at this value until $\delta=510^{-6}$.By cons, with the approach developed using wavelets, we note that the compression ratio rapidly from zero to $75.8 \%$ when $\delta$ exceeds $510^{-8}$.

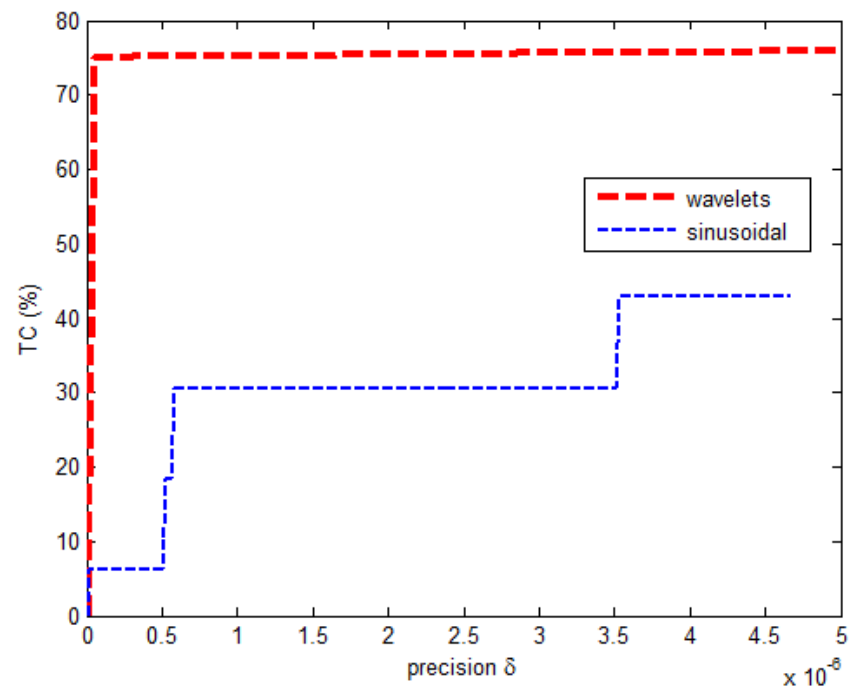

Figure 6.Compression ratio as a function of accuracy for different test functions.

Figure 7 represents the error of the input impedance as a function of compression ratio for both types of test functions. The performances obtained with two test functions bases are not identical. Indeed, for the simulation of the rectangular planar antenna, it is possible to achieve a compression ratio of $75.2 \%$ for an error on the input impedance of which does not exceed $1 \%$.

We also compared the behavior of the relative error of the reflection coefficient as a function of the accuracy $\delta$. We see from Figure 5 that the error rapidly changes from $0 \%$ to $23 \%$ when using sinusoidal functions. For the wavelet basis, this error stabilizes at $3.8 \%$ for an accuracy of up to $510^{-8}$.

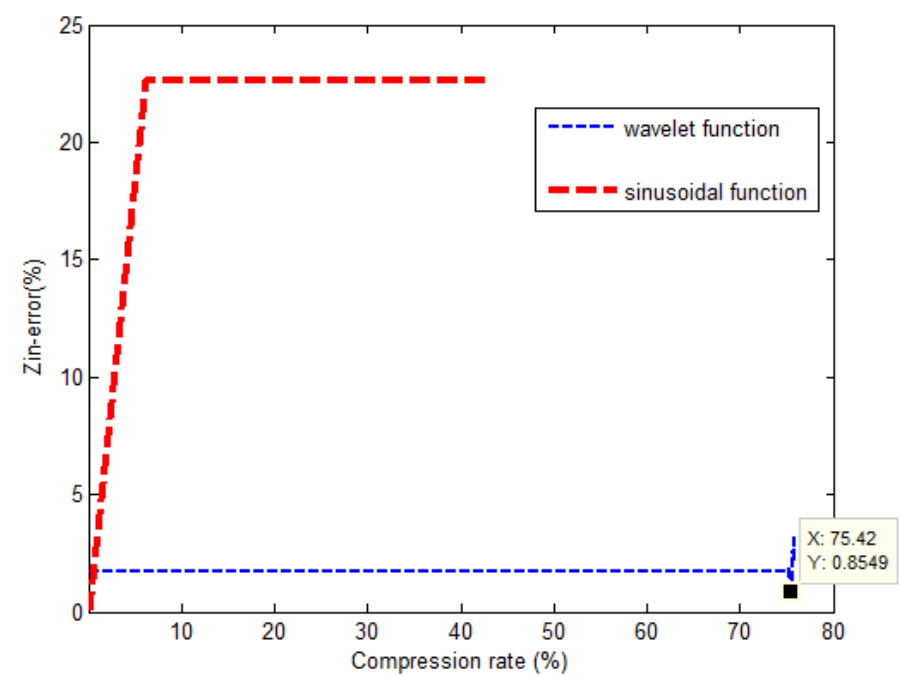

Figure 7.Error of the input impedance as a function of compression ratio for different test functions. 


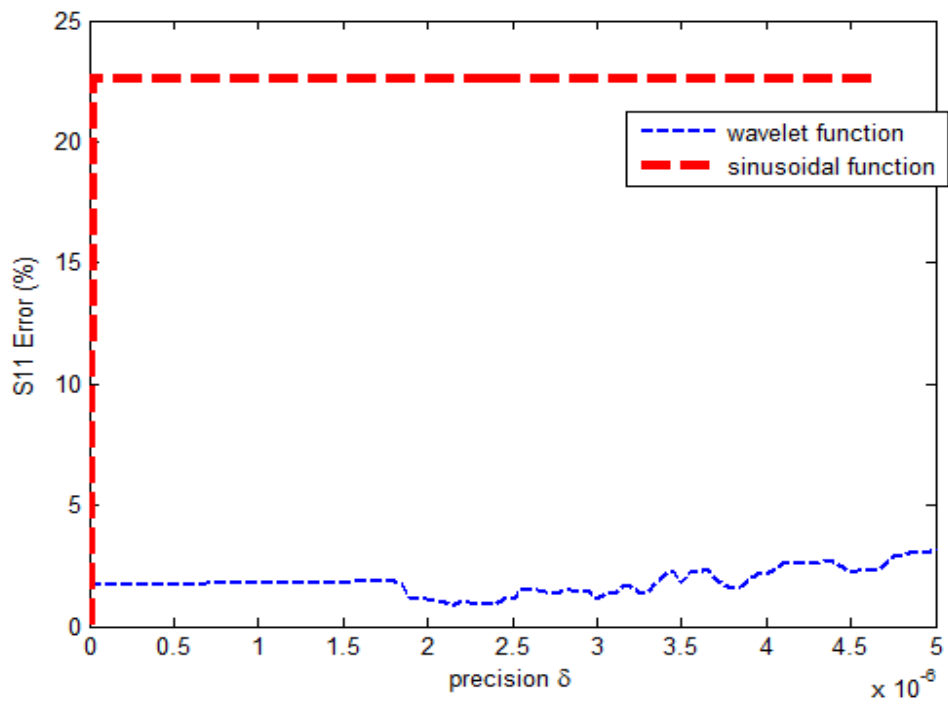

Figure 8. Error of the reflection coefficient as a function of compression ratio for different test functions.

Figure 9 represents the frequency as a function of the input impedance for different compression ratios. The results obtained at zero compression rates are consistent with those obtained with Sonnet software. For a relatively high compression ratio $(82 \%)$ the error becomes very important.

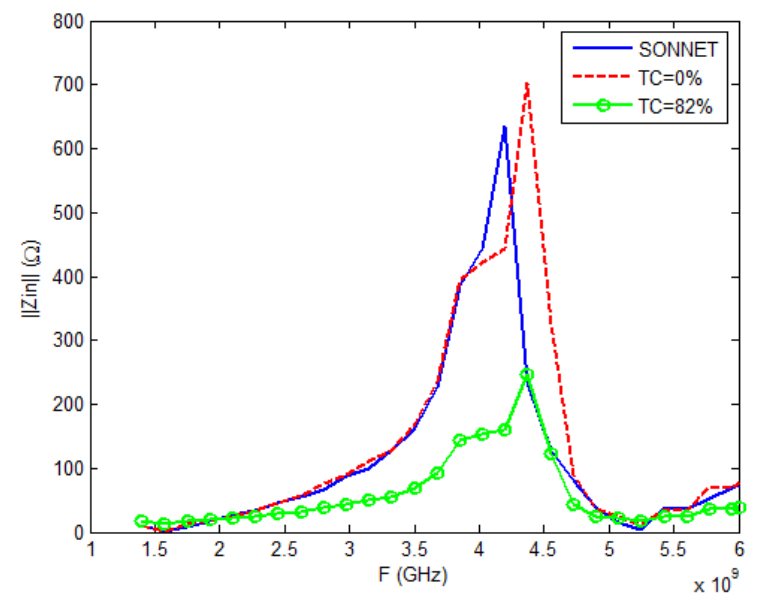

Figure 9.Variation of input impedance with frequency for different compression ratio.

\section{Gain in storage memory cost and computational time}

We highlight in this paragraph the benefits of using wavelet trial functions in storage memory cost and reducing computational time compared to traditional MoM method. Indeed, the computational time needed by MoM is as [9]:

$$
T_{M o M}=A+B P+C P^{2}+D P^{3}
$$

$P$ : unknowns' number. $A, B, C$ and $D$ are constants independent of $P$.

Aaccounts for the simulation set-up time. The meshing of the structure leads to the linear term $B P$. The filling of the system matrix is responsible for the quadratic term, and solving the matrix equation for the cubic term. The values of $A, B, C$ and $D$ depend on the problem at hand. The number of operations needed by $\operatorname{MoM} N_{o p-M o M}$ can is evaluated using:

$$
N_{o p-M o M} \approx O\left(P^{3}\right)
$$

To show the gain of the MR-GEC compared to the MoM, we need to evaluate the cost of the new approach required to compute the input impedance. 
The parameters used are the following:

- $T_{s}$ : Computational time of $\left\langle g_{p} \mid f_{n}\right\rangle$.

- $\quad T_{c}$ : Computational time of multiplication or addition.

- $\quad T_{i}(q)$ : Time inversing of a square matri including $\mathrm{q}^{2}$ elements.

- $\quad P$ : Number of test functions characterizing the structure.

- $\quad N$ : Number of waveguide modes.

The time used by the MoM-GEC to compute the input impedance of a planar microstrip structure is as:

$$
T_{M_{O M} G G E C}=N \times P \times T_{s}+\left[(N+1) \times P^{2}+P\right] \times T_{c}+T_{i}(P)+\delta
$$

So, in the MoM-GEC, the number of operations $N_{o p-M_{-} M_{-} G E C}$ can be expressed as:

$$
N_{\text {op }-M o M_{-} G E C} \approx O\left(P^{2}\right)
$$

We can deduct that $N_{o p-M o M_{-} G E C} \ll N_{o p-M o M}$ chiefly when studying large structure with significant number of test functions. This reduction in number of operations leads then to a reduction in the computational time. Obviously, in MoM-GEC, the same computational time in the impedance matrix fill in is needed as the conventional moment method since a direct scheme based on a standard numerical integration is used to compute each element of the matrix and the wavelet functions, which have localized supports, must be computed and stored.

Fortunately, we can take advantage of MALLAT's algorithm when using of wavelets as trial functions. In this paper, an indirect implementation scheme is used to improve significantly the cost of the matrix fill in. In the MoM_GEC method, to compute all matrix elements, we have to compute $=N \times P$ times the inner product $\left\langle g_{p} \mid \bar{f}_{n}\right\rangle$ for $\mathrm{N}$ modes in the modal basis and $\mathrm{P}$ different test functions.

However, according to Eq.20, we need only to calculate $f_{m, \phi_{s(+)+1, k}}$ which can be approximated by applying the one point quadrature rule.

At the finest resolution level $(s(+)+1)$, for each value of $m \in[1, N]$, the vector of $P=2^{s(+)+1}$ coefficients $\left(f_{m, \phi_{s(+)+1, k}} ; k=0,1, \ldots, 2^{s(+)+1}-1\right)$, is filtered by $h_{k}$ and $g_{k}$ employing $2 L P$ multiplications and $2 L(P-1)$ additions [21], where $\mathrm{L}$ is the filter length.

The number of required operations is divided by two with regard to the previous stage, due to the data decimation.

Therefore the total number of multiplications used to compute all scalar products $\left\langle g_{p} \mid f_{n}\right\rangle$ is $N_{\text {op_mult }}=$ $2 L N\left(P+\frac{P}{2}+\frac{P}{4}+\cdots+\frac{P}{2^{s(-)}}=4 L N P\left(1-2^{1-s(-)}\right)\right.$.

The total number of additions used to compute all scalar products is $N_{\text {op_add }}=4 L N(P-1)\left(1-2^{1-s(-)}\right)$.

Using periodic wavelets and GEC modeling, the computational time required by MR-GEC to solve a proposed problem is as:

$$
T_{M R_{-} G E C}=\left[(N+1) \times P^{2}+P+N_{o p \_m u l t}+N_{\text {op_add }}\right] \times T_{c}+T_{i}(P)+\delta
$$

In the adopted approach, after applying a threshold and by discarding all elements that are smaller than a predetermined threshold $\delta$, the impedance matrix will be converted into a very sparse matrix. Then, the use of wavelets as trial functions in the MoM_GEC method produces a sparse impedance matrix which may be solved rapidly thanks to the existence of several efficient techniques.

For such problems, wavelets can be used to obtain a solution in $O(P \log P)$ operations, where $P$ is the number of unknowns [21]. This is in contrast with a cost of $O\left(P^{3}\right)$ for a dense matrix inversion or $O\left(P^{2}\right)$ per dense matrix-vector multiply in an iterative solution such as conjugate-gradient.

Table. 2 compares the computation time required to calculate the input impedance for the test functions of wavelet type and sinusoidal type.

The comparison of the computing time of simulation obtained by using this technique with sinusoidaland wavelet test functions is shown in table 3 .

There is a noticeable improvement in the required computing time for calculating the input impedance in our proposed method despite the relatively high number of wavelets functions. For instance at the frequency of $4.9 \mathrm{GHz}$, the computing time in a $2.6 \mathrm{GHz}$ Pentium IV processor with 2 Go of RAM is about 29.81 minutes for MR_GEC and 157.56minutes for MoM_GEC with sinusoidal test functions.

At this point, the CPU time by our technique represents $18.92 \%$ of which obtained by sinusoidal functions.This performance obtained with the wavelet test functions can be explained in part by using a fast algorithm (MALLLAT algorithm) for the computation of inner products and other hand by the rapid convergence in number of function s modes.

Table.2.Comparison of CPU time.

\begin{tabular}{|l|l|l|l|}
\hline Trial functions & CPU time (minutes) & $\begin{array}{l}\text { Number of TE and TM } \\
\text { modes at convergence }\end{array}$ & $\begin{array}{l}\text { Number of trial functions } \\
\text { at convergence }\end{array}$ \\
\hline
\end{tabular}


An Improved MOM-GEC Method for Fast and Accurate Analysis of 2-D Planar Structures in....

\begin{tabular}{|l|l|l|l|}
\hline sinusoial & 157.56 & 90000 & 33 \\
\hline wavelets & 29.81 & 40000 & 512 \\
\hline
\end{tabular}

Table 3shows the memory space required to store matrices for both types of test functions. After compression, and considering the sparse matrix, the memory space required for storing the matrix in the case of the wavelet is reduced compared to the use of sinusoidal functions. A gain after compression memory space reaches $63.72 \%$ using the wavelets, and not more than $30.4 \%$ in the case of sinusoidal test functions.

Table 3.Memory space required for storage impedances matrices at $\mathrm{F}=4.9 \mathrm{GHz}$.

\begin{tabular}{|l|l|l|l|l|}
\hline \multirow{2}{*}{} & \multicolumn{2}{|l|}{ Storage space memory (KO) } & \multirow{2}{*}{ Gain (\%) } \\
\cline { 2 - 4 } & Before compression & \multicolumn{2}{|l|}{ After compression } & \\
\hline Trial functions & Dense & TC $\mathbf{\%})$ & sparse & \\
\hline sinusoïdals & 4096 & 45.91 & 2851 & 30.4 \\
\hline wavelets & 4096 & 75.87 & 1486 & 63.72 \\
\hline
\end{tabular}

\section{Conclusion}

A new formulation of the Moment Method was presented in this paper to solve electromagnetic problem. The conventional MoM algorithm was combined to the generalized equivalent circuit method to get an original formulation. This technique is based on the impedance operator that is a spatial- spectral operator allowing an easy transition from spectral to spatial domain. To obtain a sparse matrix, the multiresolution wavelet basis functions are used in this paper. This method converges to the solution in a small number of iterations. The study of 2D planar microstrip structure presenting a step discontinuity is given to illustrate the properties of this method.In conclusion, we would like to highlight that MR-GEC analysis successfully removes the complexity of the proposed problem and it will be used to study more complex structures like multilayered patch antenna that open various areas of research .

\section{References}

[1]. H. Aubert and H. Baudrand, "L'Electromagnétisme par les schémas équivalents, “ Cepaduès Editions 2003

[2]. H. Baudrand," Introduction au calcul des circuits microondes," CEPADUES Ed., ENSEEIHT, Toulouse, 1994.

[3]. B. Z. Steinberg and Y. Leviatan, "On the use of wavelet expansions in the method of moments, "IEEE Trans. Antennas and Propag. Vol $41 \mathrm{~N}^{\circ} 5$, pp. 610-619, 1993.

[4]. X. Zhu, T. Sogaru and L. Carin, "Three-dimensional biorthogonalmultiresolution time-domain method and its application to electromagnetic scattering problems, "IEEE Trans. Antennas and Propag.Vol 51, N5, pp. 1085-1092, May 2003.

[5]. C.K.CHUI, and E. QUAK,"Wavelets on a bounded interval," in BRAESS, D., and SCHUMAKER, C.L. (eds.): 'Numerical methods of approximation theory', Vol. 9, pp. 53-75, 1992.

[6]. Z. Xiang and Y. Lu, "An effective wavelet matrix transform approach for efficient solutions of electromagnetic integral equations," IEEE Trans.AntennasPropagat., vol. 45, Aug. 1997.

[7]. Vardaxoglou, J. C., Frequency Selective Surfaces, Analysis and Design, John Wiley and Sons, 1997.

[8]. Mekkioui, Z. and H. Baudrand, "A full-wave analysis of uniform microstrip leaky-wave antenna with arbitrary metallic strips," Electromagnetics, Vol. 28, No. 4, 296-314, 2008

[9]. R. F. Harrington, "Field Computation by Moment Methods, " Wiley-IEEE Press, April 1993.

[10]. Zhou, P. B., Numerical Analysis of Electromagnetic Field, 1993.

[11]. X. Chang and L. Tsang, "Fast and Broadband Modeling Method for Multiple Vias with Irregular Antipad in Arbitrarily Shaped Power/Ground Planes in 3-D IC and Packaging Based on Generalized Foldy--Lax Equations", IEEE Transactions on Components, Packaging and Manufacturing Technology, vol. 4, pp.685-696, 2014.

[12]. L. Tsang and S. Huang, "Modeling of Vias Sharing the Same Antipad in Planar Waveguide with Boundary Integral Equation and Group T Matrix Method", Progress In Electromagnetics Research, Vol. 152, pp. 105-125, 2015.

[13]. R. Loison, R. Gillard, J. Citerne, and G. Piton, "Application of the wavelet transform for the fast computation of a linear array of printed antennas," in European Microwave Conf., Vol. 2, Amsterdam, The Netherlands, pp. 301-304, 1998.

[14]. G. Oberschmidt and A. F. Jacob, "Accelerated simulation of planar circuits by means ofwavelets," in European Microwave Conf., Amsterdam, The Netherlands, pp. 305-310, 1998.

[15]. Daubechies, "Ten lectures on wavelets, "Society for Applied Mathematics Philadelphia, Pennsylvania 1992.

[16]. Pan GW., "Wavelets in electromagnetics and device modeling, "New York: Wiley; 2003.

[17]. B. Z. Steinberg and Y. Leviatan, "On the use of wavelet expansions in the method of moments, "IEEE Trans. Antennas and Propag. Vol $41 \mathrm{~N}^{\circ} 5$, pp. 610-619, 1993.

[18]. T.C. edwards, "Foundations for microstrip circuit design", NewYork, wiley 1981, secs 5.8 - 5.13.

[19]. Aguili, T., "Modélisation des composants S. H. F planaires par la méthode des circuits équivalents généralisés," Thesis, National Engineering School of Tunis ENIT, May 2000.

[20]. R. Loison, "Utilisation de l'analyse multirésolution dans la méthode des moments. Application à la modélisation de réseaux d'antennes imprimées, "Phdthesis, National Institute of Applied Sciences, Jan. 2000.

[21]. G. Beylkin, R. R. Coifmanet V. Rokhlin, "Fast wavelet transforms and numerical algorithms-I", Yale Univ. Tech. Rep. YALEU/DCS/RR-696, Aug. 1989, Commun. Pure Appl. Math., Vol. XLIV, pp. 141-183, 1991. 